\section{Marine Fish Egg Hydration Is Aquaporin-Mediated}

\author{
Mercedes Fabra, ${ }^{1}$ Demetrio Raldúa, ${ }^{1}$ Deborah M. Power, ${ }^{2}$ \\ Peter M. T. Deen, ${ }^{3}$ Joan Cerdà ${ }^{1 *}$
}

The oocytes of marine pelagic teleosts swell abruptly by hydration during the reinitiation of meiosis (oocyte maturation) before ovulation. This process renders the eggs and early embryos buoyant in sea water, allowing their survival and dispersal in the ocean $(1,2)$. The major osmotic effectors for water uptake are free amino acids derived from the proteolysis of the oocyte major yolk proteins (3), but the membrane-associated mechanisms involved remain unknown.

The time course of oocyte hydration in vitro in the marine teleost gilthead sea bream (Sparus aurata) revealed a progressive swelling of the oocyte, which appeared to accelerate within $\sim 2$ hours after the complete proteolysis of yolk proteins (fig. S1). To investigate whether molecular water channels or aquaporins (AQPs) were involved in this process, we performed reverse transcription-polymerase chain reaction (RT-PCR) to obtain AQP cDNAs from ovarian tissue. We isolated a cDNA that had a deduced amino acid sequence with six potential transmembrane domains and two asparagineproline-alanine motifs, the signature of the
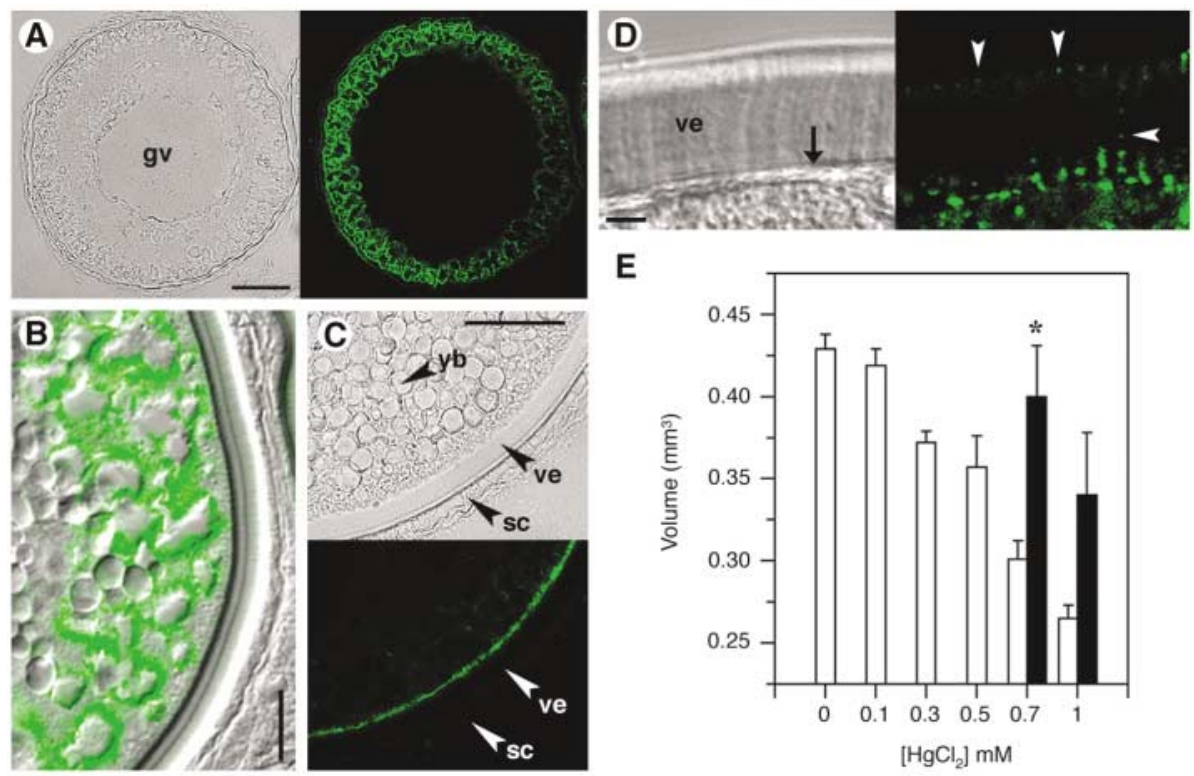

AQP superfamily (4). Heterologous expression in Xenopus laevis oocytes revealed that the encoded protein, like mammalian aquaporin-1 (AQP1) (5), was selectively permeable for water, and that its permeability could be inhibited by $\mathrm{HgCl}_{2}$ and recovered by $\beta$ mercaptoethanol ( $\beta$ ME) (fig. S2A). However, its deduced amino acid sequence showed low identity with other vertebrate AQP1 orthologs (45 to $54 \%$ ), and its mRNA was detected predominantly in the ovary (fig. S2B). On the basis of these features, we concluded that this cDNA encodes a paralog, which we named the $S$. aurata AQP1 of the ovary (SaAQP1o).

To elucidate the relationship between SaAQP1o and vertebrate AQP1-like water channels, we cloned additional sequences. In the sea bream, we identified another cDNA, the deduced protein of which was $60 \%$ identical to that of SaAQP1o, but it shared higher identity to mammalian AQP1 (57 to 59\%) and was ubiquitously expressed (fig. S2B). Phylogenetic analysis revealed that SaAQP1o, together with other AQP1-like channels found in the ovaries of some fish, fall into a distant and separate

\section{$\mathrm{E}$}

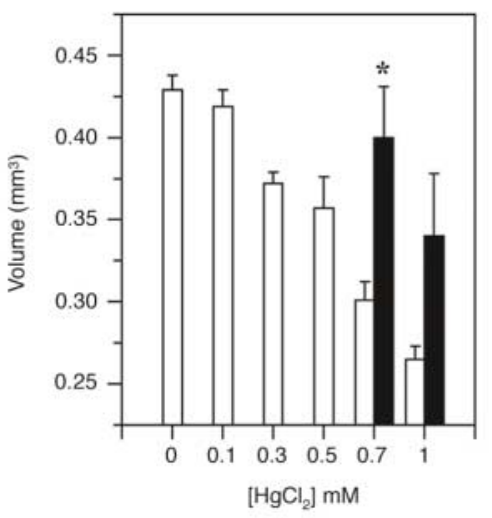

Fig. 1. (A to D) Bright-field and immunofluorescent images of SaAQP1o immunolocalization. (A) An early vitellogenic oocyte. (B) A late vitellogenic oocyte. (C) A fully grown oocyte. (D) SaAQP1o staining at the oocyte microvilli. The arrow and arrowheads indicate the plasma membrane and the microvilli, respectively. gv, germinal vesicle; yb, yolk bodies; ve, vitelline envelope; sc, somatic cells. Scale bars, (A) and (C) $50 \mu \mathrm{m}$; (B) $20 \mu \mathrm{m}$; (D) $5 \mu \mathrm{m}$. (E) Mercury inhibition of oocyte hydration in vitro (open bars) and recovery by $\beta M E$ (solid bars). Means \pm SEM are shown; *, $P<0.05$. group, suggesting the fast evolution of a unique subfamily of SaAQP1o-related channels in teleosts (fig. S2C and supporting online text).

SaAQP1o peptides were localized in the cortical cytoplasm of early vitellogenic oocytes (Fig. 1A). As oocytes developed, SaAQP1o was translocated toward a more peripheral area (Fig. 1B), whereas in fully grown oocytes, it concentrated below the plasma membrane (Fig. 1C). During oocyte maturation, SaAQP1o was further translocated into the oocyte microvilli (Fig. 1D), where it can potentially mediate water influx into the oocyte. To assess the functional relevance of these findings, follicleenclosed oocytes undergoing oocyte hydration in vitro were treated with $\mathrm{HgCl}_{2}$, which inhibited hydration in a dose-dependent and reversible manner (Fig. 1E). These results paralleled the permeability properties of SaAQP1o expressed in $X$. laevis oocytes and thus provide strong support for a role of SaAQP1o in mediating sea bream oocyte hydration.

In conclusion, we found that SaAQP1o belongs to a subfamily of AQP1-like channels evolved in teleosts, which are likely derived from a common AQP1-like ancestral gene. Although SaAQP1o orthologs in other teleosts might have other, yet-unknown osmoregulatory functions, our results reveal that fish oocyte hydration is based on the interplay between protein hydrolysis and SaAQP1o, indicating a specialized physiological role for this AQP.

\section{References and Notes}

1. P. J. Mellinger, Annee Biol. 33, 117 (1994).

2. B. Ådlandsvik, S. Coombs, S. Sundby, G. Temple, Fish. Res. 50, 59 (2001)

3. K. Selman, R. A. Wallace, J. Cerdà, J. Exp. Zool. 290, 265 (2001).

4. P. Agre et al., J. Physiol. 542, 3 (2002).

5. G. M. Preston, T. P. Carroll, W. B. Guggino, P. Agre, Science 256, 385 (1992).

6. We thank E. Lubzens, W. W. de Jong, and F. Piferrer for critical reading of the manuscript. Supported by the Spanish Ministry of Science and Technology (MCyT) and European Commission grants (J.C.) and by a fellowship from the Catalan government (M.F.). This work is dedicated to Prof. R. A. Wallace, a pioneer in the investigation of oocyte hydration in fish, who inspired and encouraged this research.

Supporting Online Material

www.sciencemag.org/cgi/content/full/307/5709/545/ DC1

Materials and Methods

SOM Text

Figs. S1 and S2

References and Notes

12 October 2004; accepted 5 November 2004 10.1126/science. 1106305

${ }^{1}$ Center of Aquaculture-Institut de Recerca i Tecnologia Agroalimentaries, Tarragona, Spain, and Reference Center in Aquaculture, Barcelona, Spain. ${ }^{2}$ Center of Marine Sciences, Universidade do Algarve, Faro, Portugal. ${ }^{3}$ Department of Physiology, Radboud University of Nijmegen Medical Center, Nijmegen, Netherlands.

*To whom correspondence should be addressed. E-mail: jcerda@icm.csic.es 


\section{Supporting Online Material}

\section{Materials and methods}

Fish. Adult gilthead seabream were obtained from local fishermen, and Senegal sole (Solea senegalensis) and sea bass (Dicentrarchus labrax) were purchased from commercial fish farms. Wild-type zebrafish (Danio rerio) were obtained from a local pet store. Fish were sacrificed by stunning and decapitation, and the ovary and other tissues were used for isolation of ovarian follicles, RNA extraction and immunocytochemistry. All procedures for the sampling of fish and sacrifice were approved by the Ethical Committee from IRTA (Spain).

\section{Water content measurement, culture of ovarian follicles in vitro, and SDS-PAGE of yolk}

proteins. Seabream gonads were placed in 75\% Leivovitz L-15 culture medium with L-glutamine (Sigma), and fully-grown, follicle-enclosed oocytes were manually isolated as described (S1). Water content of fully-grown and mature oocytes and eggs $(n=200)$ was measured gravimetrically to constant weight at $60^{\circ} \mathrm{C}$. Water content increased from $64.8 \%$ in fully-grown oocytes to $91.5 \%$ in mature oocytes and eggs (\% in weight). For induction of oocyte maturation and hydration in vitro, fully-grown ovarian follicles were incubated in culture medium with $0.1 \mu \mathrm{g} \mathrm{m}{ }^{1}$ of the maturation-inducing steroid (MIS) $17 \alpha, 20 \beta$-dihydroxy-4-pregnen-3-one up to $48 \mathrm{~h}$ at $18^{\circ} \mathrm{C}$. To evaluate the effect of mercury on oocyte hydration, groups of follicles undergoing maturation in vitro ("stippled" stages), not fully hydrated, were incubated with $\mathrm{HgCl}_{2}$ for 1 h. The effect of $\mathrm{HgCl}_{2}$ was reversed by immediate post-incubation of $\mathrm{HgCl}_{2}$-treated oocytes with $5 \mathrm{mM} \beta$-mercaptoethanol ( $\beta \mathrm{ME}$ ) for $15 \mathrm{~min}$. Changes in oocyte volume were calculated from the oocyte diameter measured with an ocular stereomicroscope to the nearest $0.01 \mathrm{~mm}$ until full hydration was observed. Yolk 
proteins extracts from follicles undergoing maturation and hydration were analyzed by SDS-PAGE as described $(S 2)$.

Cloning of fish aquaporins and phylogenetic analysis. Full-length complementary DNAs (cDNAs) of SaAQP1o and SsAQP were cloned from total RNA extracted from the ovary (Qiagen) using RT-PCR and degenerate primers corresponding to the highly conserved regions of the major intrinsic protein (MIP) gene family (S3). Rapid amplification of cDNA ends (RACE, Gibco) was further employed to isolate the 5' and 3' ends, followed by a final amplification of the full-lenght cDNAs with a high-fidelity polymerase ( $P w o$, Roche). Full-length DrAQP1 and SaAQP1 cDNAs were isolated by RT-PCR using a deposited expressed sequence tag (GenBank accession number BQ783573), and by screening a kidney cDNA library (S4), respectively. The partial DIAQP1 cDNA was cloned from kidney total RNA using the forward degenerate primer initially employed and a reverse primer (AUAP) specific for the adapter region of the oligo d(T) used for cDNA synthesis (Gibco). Multiple amino acid sequence alignments and phylogenetic analyses using the neighbour-joining method were performed with the Clustal W multiple sequence alignment program (www.ebi.ac.uk/clustalw) and drawn with the Phylodendron application (www.es.embnet.org/Doc/phylodendron/treeprint-form.html).

Gene expresion analysis. The abundance of SaAQP1o and SaAQP1 transcripts in different adult tissues was determined by RT-PCR on oligo dT-synthesised cDNAs using PCR cycles (32, and 30, respectively) that generated half-maximal amounts of PCR product (not shown). The forward and reverse oligonucleotide primers for SaAQP1o were: 5' -GGCGGCTCTTATCTACGATTT-3' and 5' -TGAAAGCTTTTCTGCAACTCA-3', respectively, whereas for SaAQP1 these were: 5' GGCTCTCACGTACGATTTCC-3', and 5'-TCTGTGTGGGACTATTTTGACG-3'. The PCR for $\beta$-actin (24 cycles) was performed to control the variation in mRNA concentration in the RT reaction using the following forward and reverse primers, respectively: 5' - 
GCAAAACAACCACACCACAC-3', and 5'-GGCGAATACGTGGTCTGAACA-3' (GenBank accession number X89920).

Functional expression in Xenopus laevis oocytes. Complementary RNA production (cRNA), expression of SaAQP1o in X. laevis oocytes and osmotic water permeability $\left(P_{\mathrm{f}}\right)$ measurements, in the presence or absence of $\mathrm{HgCl}_{2}$ and $\beta \mathrm{ME}$, were performed as described (S5).

Antibody production and immunofluorescence. A peptide was synthesized corresponding to the C-terminal 20 amino acids of SaAQP1o (PREGNSSPGPSQGPSQWPKH) and injected into rabbits (Cambridge Research Biochemicals). The antisera were affinity-purified on thiopropyl sepharose 6B coupled to the synthetic peptide. Immunofluorescence on paraplast (Sigma) embedded tissue sections was carried out on tissues fixed either in $4 \%$ paraformaldehyde or Bouin's without acetic using standard protocols. The anti-SaAQP1o antibody (1:300-500) was applied for $1 \mathrm{~h}$ at room temperature or overnight at $4^{\circ} \mathrm{C}$. Using the pre-immune serum or pre-incubation of the antibody with the synthetic peptide for $1 \mathrm{~h}$ at $37^{\circ} \mathrm{C}$ previous to its application onto the sections did not reveal any staining (not shown), which demonstrated the specificity of the signals. Pictures were taken using a confocal laser-scanning microscope.

Data analysis. Data from in vitro incubations of sea bream oocytes and $P_{\mathrm{f}}$ values from $X$. laevis oocytes expressing SaAQP1o were statistically analyzed by the Student T-test. Differences were considered significant at $P<0.05$.

GenBank accession numbers. The teleost aquaporin sequences described in this paper have been deposited in GenBank under the accession numbers: SaAQP1o (AY626938), SaAQP1 (AY626939), S. senegalensis AQP1 (AY626941), D. labrax AQP1 (AY626940), D. rerio AQP1 (AY626937). 


\section{SOM Text}

The phylogenetic tree depicted in fig. S2C shows that the branches of ovarian AQP1o proteins are considerably larger than those of normal fish aquaporin-1 proteins. This suggests a faster evolution of the AQP1o proteins, which is often observed for proteins with a specific function (as SaAQP1o shown here). This rapid evolution might be helped by the genome duplication early in evolution in teleosts, because this allowed a fast development of one copy into a specialized function, while the old function will be maintained by the remaining aquaporin-1. During evolution, fish evolved from seawater to freshwater, but later in evolution fish are thought to have switched sometimes between seawater and fresh water (S6). This means that AQP1o proteins might have developed for generating egg buoyancy in particular fish species when they were in seawater, while this function might have been lost later with the switch of these fish species to freshwater. Therefore, in freshwater fish, in which eggs do not become buoyant, AQP1o might be still present. Indeed, catfish, which encode a SaAQP1o-related protein (lpAQP1o), is a freshwater species. In these fish, AQP1o might have a similar function, might be redundant, or might have obtained another osmoregulatory function in the ovary. In salmonids and other "primitive" freshwater teleosts, such as cyprinidontiforms and siluriforms (e.g., catfish), a continuous hydration of the yolk and/or the oocyte during meiotic maturation and ovulation, resulting in a low increase in oocyte volume (5-10\%), has been described (S7-S10). Therefore, AQP1o proteins in freshwater fish might have a similar function as in pelagic marine fish (e.g., mediating oocyte/yolk hydration), but resulting in non-buoyant eggs. 


\section{Supporting References and Notes}

S1. J. Cerdà, K. Selman, R. A. Wallace, Aquat. Living Resourc. 9, 325 (1996).

S2. K. Selman, R. A. Wallace, J. Cerdà, J. Exp. Zool. 290, 265 (2001).

S3. G. M. Preston, Meth. Mol. Biol. 69, 97 (1997).

S4. C. R. A. Santos et al., J. Exp. Biol. 207, 1217 (2004).

S5. P. M. T. Deen et al., Science 264, 92 (1994).

S6. J. A. Long, The rise of fishes. 500 Million years of evolution. Baltimore: The John Hopkins University Press, 223 p. (1995).

S7. H. P. Clemens, F. B. Grant, Zoologica 49, 193 (1964).

S8. W. T. W. Potts, P. P. Rudy, J. Exp. Biol. 50, 223 (1969).

S9. T. Iwamatsu et al., Dev. Growth Differ. 34, 173 (1992).

S10. K. Selman et al., J. Morphol. 218, 203 (1993). 


\section{Legends to the Supporting Figures}

Fig. S1. Time-course of oocyte hydration (A) and proteolysis of major yolk proteins (B) during MIS-activated meiotic maturation of seabream oocytes in vitro. (A) Representative volume changes over time of a single oocyte $(n=8)$ treated with $0.1 \mathrm{mg} \mathrm{ml} 1 \mathrm{of}$ MIS (closed circles) or ethanol vehicle (Control, open circles). (B) SDS-PAGE of follicle protein extracts (5 $\mu \mathrm{g} / \mathrm{lane})$ at the specific hydration stages ( 1 to 5) indicated in (A) (the arrowhead indicates degradation of a major yolk protein of approximately $100 \mathrm{kDa}$ ).

Fig. S2. (A) Osmotic water permeability $\left(P_{\dot{f}}\right.$, mean \pm SEM; $\left.n=9\right)$ of $X$. laevis oocytes injected with water or $1 \mathrm{ng}$ of SaAQP1o cRNA, and its inhibition and recovery by $1 \mathrm{mM} \mathrm{HgCl}$ and $5 \mathrm{mM} \beta \mathrm{ME}$, respectively. Asterisks denote significant differences $(P=0.05)$. (B) Reverse transcriptasepolymerase chain reaction (RT-PCR) analysis of SaAQP1o and SaAQP1 transcripts in adult tissues (minus indicates absence of RT during cDNA synthesis). (C) Phylogenetic relationships of vertebrate aquaporin-1 (AQP1)-like water channels. The GenBank accession numbers of the sequences used were: human (Homo sapiens; Hs; NP_000376), mouse (Mus musculus; Mm; BC007125), rat (Rattus norvegicus; Rn; NM_012778), sheep (Ovies aries; Oa; AF009037), frog (Hila japonica; Hj; AB073315), edible frog (Rana esculenta; Re; L24754), toad (Bufo marinus; Bm; AF020620), catfish (Ictalurus punctatus; Ip; CK418363), Senegal sole (S. senegalensis; Ss; AY626941), gilthead seabream (S. aurata; Sa; AY626938 and AY626939), sea bass (D. labrax; D1; AY626940), black sea bass (Centropristis striata; Cs; AY626936), puffer fish (Fugu rubripes; Fr; FRUP00000130258), Japanese eel (Anguilla japonica; Aj; AB094501), and zebrafish (D. rerio; Dr; AY626937). 
A

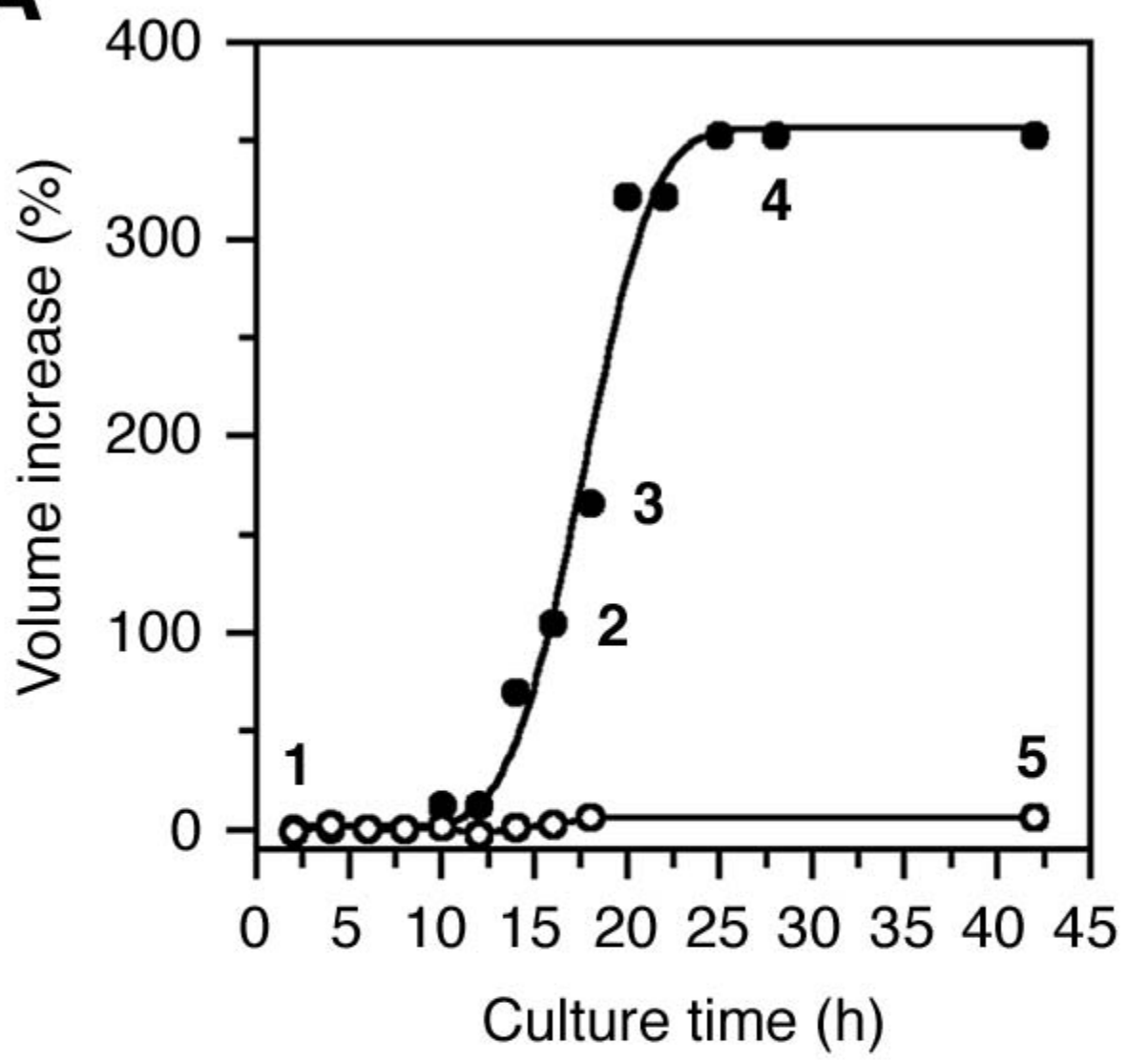

B

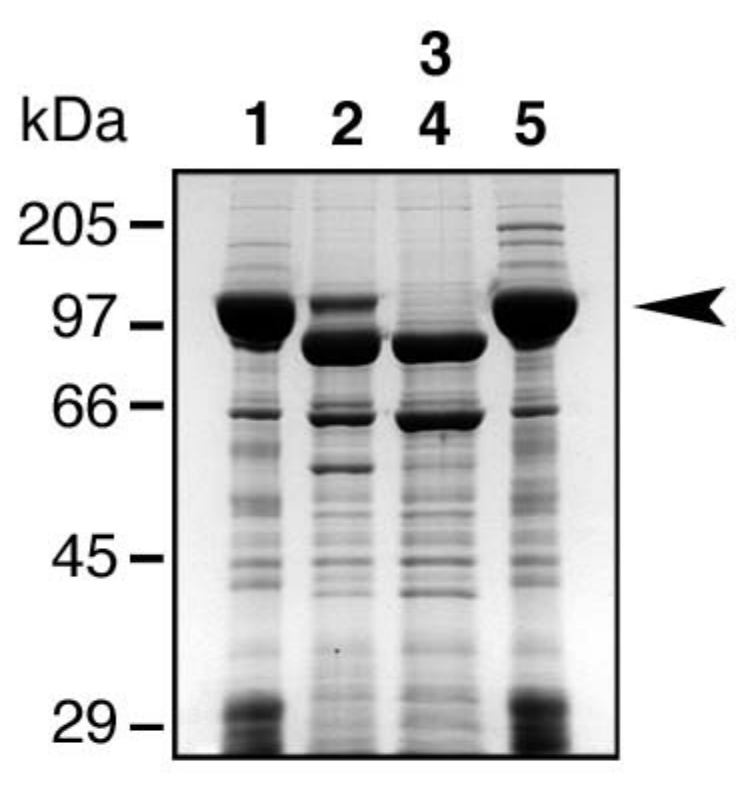

Fabra et al. Fig. S1 
A

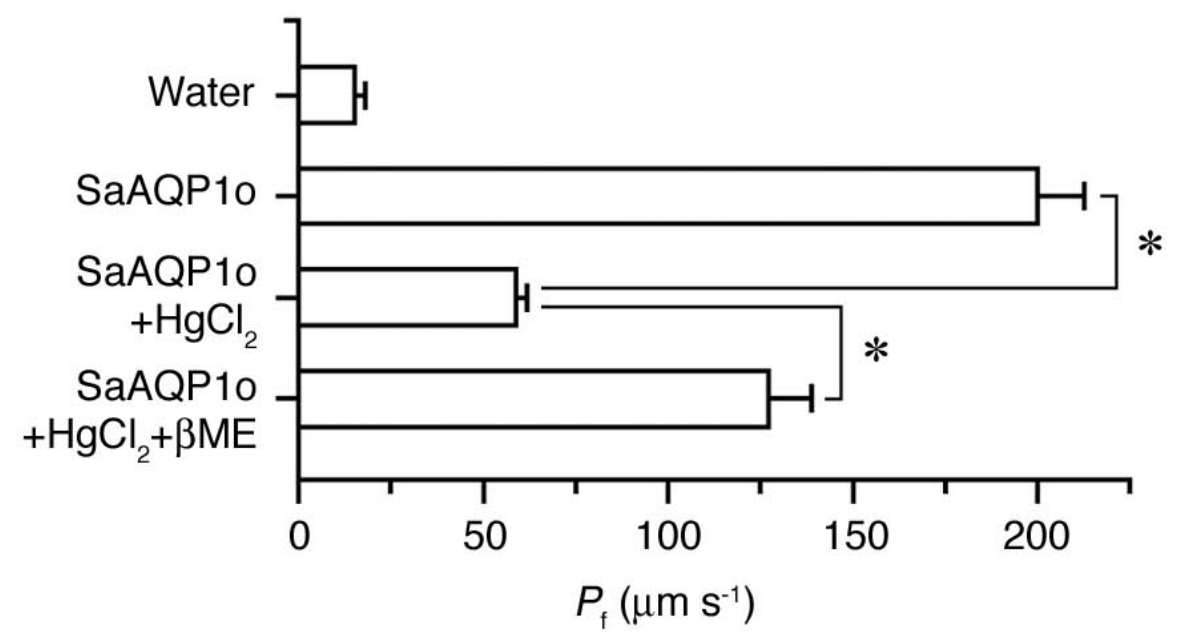

B
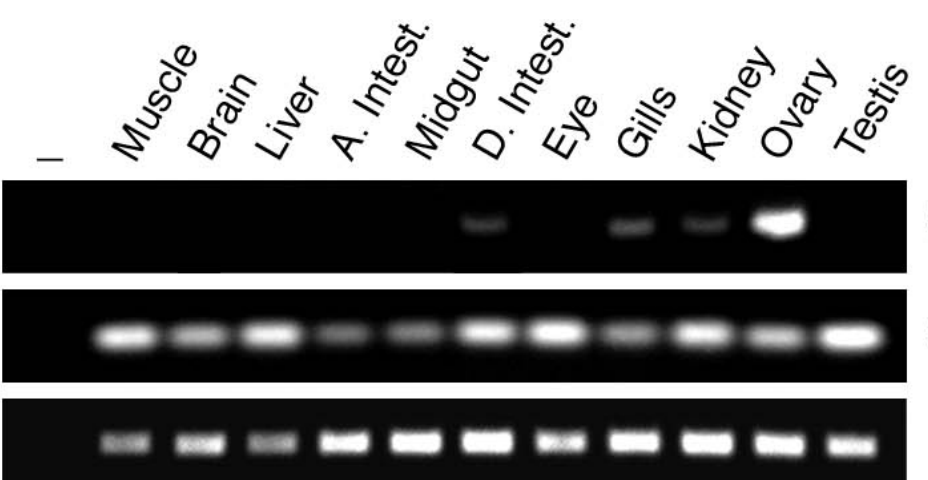

SaAQP1o

SaAQP1

$\beta$-actin
C

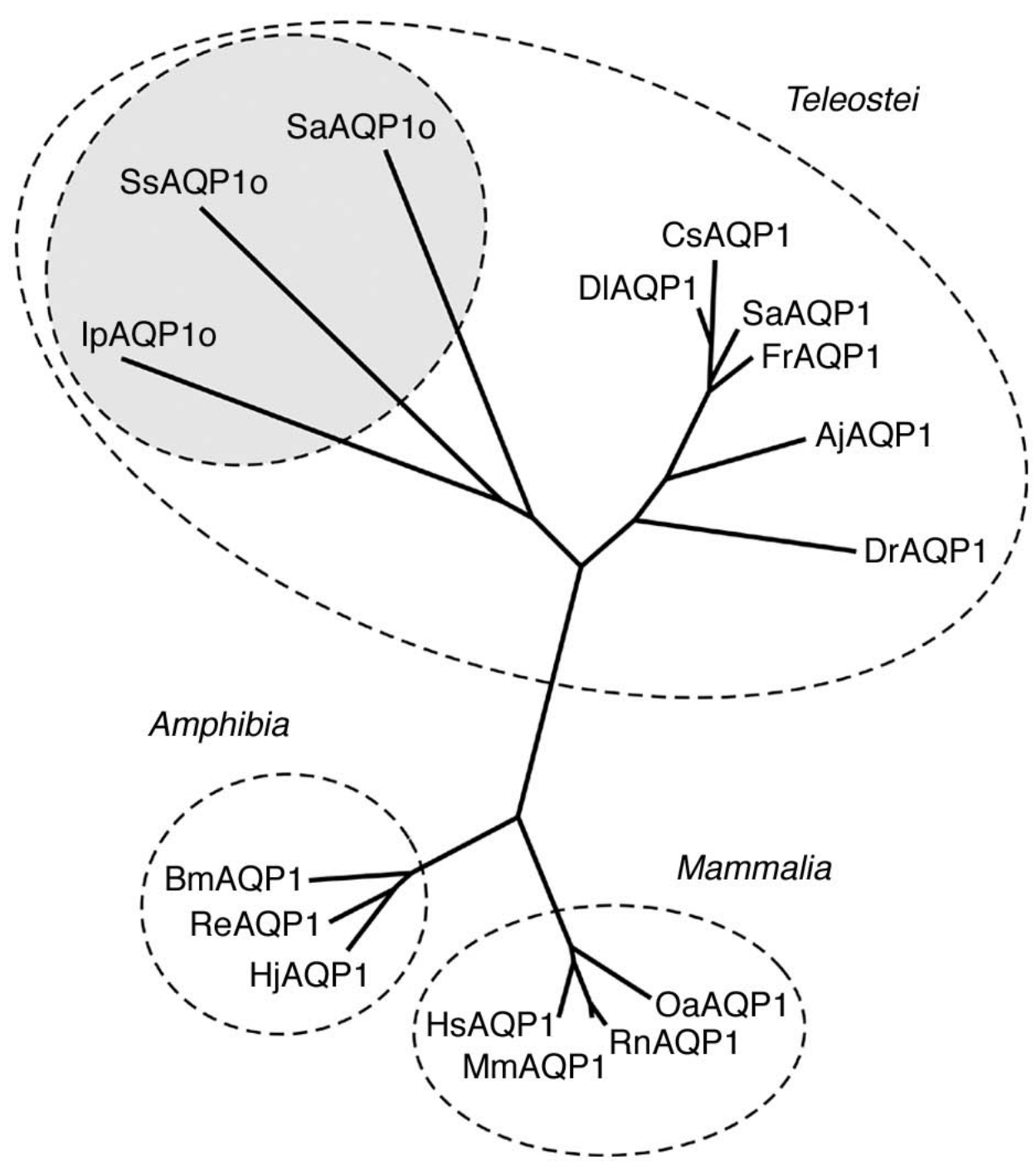

\section{Fabra et al. Fig. S2}

